\title{
MULTIPLE FRACTIONAL PART INTEGRALS AND EULER'S CONSTANT
}

\section{OVIDIU FURDUI}

Received 27 December, 2013

Abstract. The paper is about calculating multiple fractional part integrals of the form

$$
\int_{0}^{1} \int_{0}^{1}(x+y)^{k}\left\{\frac{1}{x+y}\right\}^{p} d x d y \quad \text { and } \int_{0}^{1} \int_{0}^{1} \int_{0}^{1}\left\{\frac{1}{x+y+z}\right\}^{m} d x d y d z
$$

where $\{x\}$ denotes the fractional part of $x$ and $k, m$ and $p$ are nonnegative integers. We show that these integrals can be expressed as series involving products of Riemann zeta function values and some binomial coefficients. We obtain, as particular cases of our results, new integral representations of Euler's constant as double and triple symmetric fractional part integrals.

2010 Mathematics Subject Classification: 26A42; 26B15; 40A30

Keywords: the Euler-Mascheroni constant, harmonic numbers, fractional part integrals, Riemann zeta function

\section{INTRODUCTION}

Let $m, p$ and $k$ be nonnegative integers and let $D_{k, p}$ and $I_{m}$ denote the double and triple symmetric integrals

$$
D_{k, p}=\int_{0}^{1} \int_{0}^{1}(x+y)^{k}\left\{\frac{1}{x+y}\right\}^{p} d x d y
$$

and

$$
I_{m}=\int_{0}^{1} \int_{0}^{1} \int_{0}^{1}\left\{\frac{1}{x+y+z}\right\}^{m} d x d y d z
$$

where $\{x\}$ denotes the fractional part of $x$ and is therefore related to the floor function by $\{x\}=x-\lfloor x\rfloor$. The integral $I_{m}$ can be viewed as a three dimensional version of Havil's integral $\int_{0}^{1}\{1 / x\} d x=1-\gamma$ ([3, pp. 109-111]), while the integral $D_{k, m}$ generalizes the double symmetric integral

$$
\int_{0}^{1} \int_{0}^{1}\left\{\frac{1}{x+y}\right\} d x d y
$$


proposed by Furdui as a problem in [1] and solved by Qin in [4]. We also consider the multiple integral

$$
M_{m}^{n}=\int_{0}^{1} \cdots \int_{0}^{1}\left(x_{1}+x_{2}+\cdots+x_{n}\right)^{m}\left\{\frac{1}{x_{1}+\cdots+x_{n}}\right\} d x_{1} \cdots d x_{n},
$$

where $n \geq 2$ and $m \geq 0$ are integers, and we show that this integral can be calculated via a recurrence formula.

The goal of this paper is to calculate, in closed form, the three classes of integrals given above and to prove that these integrals can be expressed in terms of series involving products of Riemann zeta function values and some binomial coefficients. We obtain, as particular cases of our results, new integral representations of the Euler-Mascheroni constant in terms of double symmetric fractional part integrals. The organization of the paper is as follows: in the next section we calculate the double integrals $D_{k, p}$, in section 3 we concentrate on the evaluation of the integral $I_{m}$ and in section 4 we evaluate the integral $M_{m}^{n}$ via a recurrence formula.

Before we give the main results of this paper we need to collect a result from [2], Theorem 1 below, which is about calculating the special class of single fractional part integrals $V_{k, m}$ defined by

$$
V_{k, m}=\int_{0}^{1} x^{m}\left\{\frac{1}{x}\right\}^{k} d x
$$

where $k$ and $m$ are nonnegative integers.

Theorem 1. Let $m \geq 0$ and let $k \geq 1$ be integers. Then

$$
V_{k, m}=\int_{0}^{1} x^{m}\left\{\frac{1}{x}\right\}^{k} d x=\frac{k !}{(m+1) !} \sum_{j=1}^{\infty} \frac{(m+j) !}{(k+j) !}(\zeta(m+j+1)-1) .
$$

In particular, one has as a consequence of Theorem 1, the following corollary.

Corollary 1. a) Let $m \geq 1$ be an integer. Then

$$
V_{m, m}=\int_{0}^{1}\left\{\frac{1}{x}\right\}^{m} x^{m} d x=1-\frac{\zeta(2)+\zeta(3)+\cdots+\zeta(m+1)}{m+1} .
$$

b) Let $m \geq 1$ be an integer. Then

$$
V_{m+1, m}=\int_{0}^{1} x^{m}\left\{\frac{1}{x}\right\}^{m+1} d x=H_{m+1}-\gamma-\sum_{j=2}^{m+1} \frac{\zeta(j)}{j},
$$

where $H_{m+1}$ denotes the $(m+1)$ th harmonic number.

c) Let $m \geq 0$ be an integer. Then

$$
V_{m, m+1}=\int_{0}^{1} x^{m+1}\left\{\frac{1}{x}\right\}^{m} d x=\frac{1}{2}+\frac{1}{(m+1)(m+2)}\left(\zeta(2)-\sum_{i=1}^{m+1} i \zeta(i+1)\right) .
$$


Proof. The first two parts of the corollary are proved in [2], so we need to prove only part c) of the corollary. We have, in view of Theorem 1, that

$$
\begin{aligned}
V_{m, m+1} & =\frac{m !}{(m+2) !} \sum_{j=1}^{\infty} \frac{(m+1+j) !}{(m+j) !}(\zeta(m+j+2)-1) \\
& =\frac{1}{(m+1)(m+2)} \sum_{j=1}^{\infty}(m+j+1)(\zeta(m+j+2)-1),
\end{aligned}
$$

and the result follows since $\sum_{i=1}^{\infty} i(\zeta(i+1)-1)=\zeta(2)$.

\section{A DOUBLE SYMMETRIC FRACTIONAL PART INTEGRAL}

In this section we calculate the double integral $D_{k, p}$. We prove that the evaluation of the double integral $D_{k, p}$ reduces to the calculation of the single integral $V_{k, m}$. The main result of this section is the following theorem.

Theorem 2. a) Let $D_{k, p}$ be as in (1.1) and let $k$ be a nonnegative integer. Then

$$
\begin{aligned}
D_{k, k+1} & =\int_{0}^{1} \int_{0}^{1}(x+y)^{k}\left\{\frac{1}{x+y}\right\}^{k+1} d x d y \\
& =2 \ln 2-\frac{\zeta(2)+\zeta(3)+\cdots+\zeta(k+2)}{k+2} .
\end{aligned}
$$

b) A new integral formula for Euler's constant. Let $k$ be a nonnegative integer. Then

$$
D_{k, k+2}=\int_{0}^{1} \int_{0}^{1}(x+y)^{k}\left\{\frac{1}{x+y}\right\}^{k+2} d x d y=1-\ln 2+H_{k+2}-\gamma-\sum_{i=2}^{k+2} \frac{\zeta(i)}{i} .
$$

c) If $p \neq k+1$ and $p \neq k+2$ then

$$
D_{k, p}=\frac{2^{k-p+2}-2}{k-p+1}-\frac{2^{k-p+2}-1}{k-p+2}+V_{p, k+1} .
$$

Proof. We have, based on the substitution $x+y=t$ in the inner integral, that

$$
D_{k, p}=\int_{0}^{1}\left(\int_{0}^{1}(x+y)^{k}\left\{\frac{1}{x+y}\right\}^{p} d y\right) d x=\int_{0}^{1}\left(\int_{x}^{x+1} t^{k}\left\{\frac{1}{t}\right\}^{p} d t\right) d x .
$$

We integrate by parts with

$$
f(x)=\int_{x}^{x+1} t^{k}\left\{\frac{1}{t}\right\}^{p} d t, \quad f^{\prime}(x)=(x+1)^{k}\left\{\frac{1}{x+1}\right\}^{p}-x^{k}\left\{\frac{1}{x}\right\}^{p},
$$


$g^{\prime}(x)=1, g(x)=x$, and we get that

$$
\begin{aligned}
D_{k, p}= & \left.\left(x \int_{x}^{x+1} t^{k}\left\{\frac{1}{t}\right\}^{p} d t\right)\right|_{x=0} ^{x=1} \\
& -\int_{0}^{1} x\left((x+1)^{k}\left\{\frac{1}{x+1}\right\}^{p}-x^{k}\left\{\frac{1}{x}\right\}^{p}\right) d x \\
= & \int_{1}^{2} t^{k}\left\{\frac{1}{t}\right\}^{p} d t-\int_{0}^{1} x(x+1)^{k-p} d x+\int_{0}^{1} x^{k+1}\left\{\frac{1}{x}\right\}^{p} d x \\
= & \int_{1}^{2} t^{k-p} d t-\int_{0}^{1} x(x+1)^{k-p} d x+V_{p, k+1} .
\end{aligned}
$$

We used the fact that, for $x>0$, one has that $\{1 /(1+x)\}=1 /(1+x)$. We distinguish here the following cases.

Case $p=k+1$. We have, based on (2.1) and part a) of Corollary 1, that

$$
\begin{aligned}
D_{k, k+1} & =\int_{1}^{2} \frac{d t}{t}-\int_{0}^{1} \frac{x}{x+1} d x+V_{k+1, k+1} \\
& =2 \ln 2-1+V_{k+1, k+1} \\
& =2 \ln 2-\frac{1}{k+2}(\zeta(2)+\zeta(3)+\cdots+\zeta(k+2)) .
\end{aligned}
$$

Case $p=k+2$. We have, in view of (2.1) and part b) of Corollary 1, that

$$
\begin{aligned}
D_{k, k+2} & =\int_{1}^{2} \frac{d t}{t^{2}}-\int_{0}^{1} \frac{x}{(x+1)^{2}} d x+V_{k+2, k+1} \\
& =1-\ln 2+V_{k+2, k+1} \\
& =1-\ln 2+H_{k+2}-\gamma-\sum_{i=2}^{k+2} \frac{1}{i} \zeta(i) .
\end{aligned}
$$

Case $p \neq k+1$ and $p \neq k+2$. Equality (2.1) implies that

$$
\begin{aligned}
D_{k, p} & =\left.\frac{t^{k-p+1}}{k-p+1}\right|_{t=1} ^{t=2}-\left.\frac{(x+1)^{k-p+2}}{k-p+2}\right|_{x=0} ^{x=1}+\left.\frac{(x+1)^{k-p+1}}{k-p+1}\right|_{x=0} ^{x=1}+V_{p, k+1} \\
& =\frac{2^{k-p+2}-2}{k-p+1}-\frac{2^{k-p+2}-1}{k-p+2}+V_{p, k+1},
\end{aligned}
$$

and the theorem is proved. 
Corollary 2. Let $k \geq 0$ be an integer. Then

$$
\begin{aligned}
D_{k, k} & =\int_{0}^{1} \int_{0}^{1}(x+y)^{k}\left\{\frac{1}{x+y}\right\}^{k} d x d y \\
& =1+\frac{1}{(k+1)(k+2)}\left(\zeta(2)-\sum_{i=1}^{k+1} i \zeta(i+1)\right) .
\end{aligned}
$$

Proof. We have, based on Theorem 2 with $p=k$, that $D_{k, k}=1 / 2+V_{k, k+1}$, and the result follows from part c) of Corollary 1.

In particular one has that

$$
\int_{0}^{1} \int_{0}^{1}(x+y)\left\{\frac{1}{x+y}\right\} d x d y=1-\frac{\zeta(3)}{3}
$$

and

$$
\int_{0}^{1} \int_{0}^{1}(x+y)^{2}\left\{\frac{1}{x+y}\right\}^{2} d x d y=1-\frac{\zeta(3)}{6}-\frac{\zeta(4)}{4}
$$

Corollary 3. The following equality holds

$$
\int_{0}^{1} \int_{0}^{1} x(x+y)^{k}\left\{\frac{1}{x+y}\right\}^{p} d x d y=\frac{1}{2} D_{k+1, p}
$$

Proof. We have, by symmetry reasons, that

$$
\int_{0}^{1} \int_{0}^{1} x(x+y)^{k}\left\{\frac{1}{x+y}\right\}^{p} d x d y=\int_{0}^{1} \int_{0}^{1} y(x+y)^{k}\left\{\frac{1}{x+y}\right\}^{p} d x d y,
$$

and it follows that

$$
\begin{aligned}
\int_{0}^{1} \int_{0}^{1} x(x+y)^{k}\left\{\frac{1}{x+y}\right\}^{p} d x d y & =\frac{1}{2} \int_{0}^{1} \int_{0}^{1}(x+y)^{k+1}\left\{\frac{1}{x+y}\right\}^{p} d x d y \\
& =\frac{1}{2} D_{k+1, p},
\end{aligned}
$$

and the corollary is proved.

We have that, for any nonnegative integer $k$, the following equality holds

$$
\int_{0}^{1} \int_{0}^{1} x(x+y)^{k-1}\left\{\frac{1}{x+y}\right\}^{k} d x d y=\frac{1}{2}+\frac{\zeta(2)-\sum_{i=1}^{k+1} i \zeta(i+1)}{2(k+1)(k+2)}
$$




\section{A TRIPLE HAVIL INTEGRAL}

In this section we calculate the integral $I_{m}$, which we call the triple Havil integral, and we prove that the evaluation of this class of integrals reduces to the calculation of the double integral $D_{1, m}$. The main result of this section is the following theorem.

Theorem 3. Let $I_{m}$ be as in (1.2). a) Then

$$
I_{1}=\frac{9 \ln 3}{2}-6 \ln 2-\frac{\zeta(3)}{6} \quad \text { and } \quad I_{2}=6 \ln 2-3 \ln 3-\frac{\zeta(2)+\zeta(3)}{6} .
$$

b) A cubic integral. We have

$$
I_{3}=\int_{0}^{1} \int_{0}^{1} \int_{0}^{1}\left\{\frac{1}{x+y+z}\right\}^{3} d x d y d z=\frac{\ln 3}{2}-\frac{3 \ln 2}{2}+\frac{5}{3}-\frac{\gamma}{2}-\frac{\zeta(2)}{4}-\frac{\zeta(3)}{6} .
$$

c) Let $m \geq 4$ be an integer. Then

$$
I_{m}=\frac{(7-m) 2^{2-m}+m-4-3^{3-m}}{(m-1)(m-2)(m-3)}+\frac{1}{2} D_{1, m} .
$$

Proof. We have, based on the substitution $x+y+z=t$, that

$$
\begin{aligned}
I_{m} & =\int_{0}^{1} \int_{0}^{1}\left(\int_{x+y}^{x+y+1}\left\{\frac{1}{t}\right\}^{m} d t\right) d x d y \\
& =\int_{0}^{1}\left(\int_{0}^{1}\left(\int_{x+y}^{x+y+1}\left\{\frac{1}{t}\right\}^{m} d t\right) d x\right) d y .
\end{aligned}
$$

We calculate the double inner integral by parts with

$$
f(x)=\int_{x+y}^{x+y+1}\left\{\frac{1}{t}\right\}^{m} d t, \quad f^{\prime}(x)=\left\{\frac{1}{1+x+y}\right\}^{m}-\left\{\frac{1}{x+y}\right\}^{m},
$$

$g^{\prime}(x)=1, g(x)=x$, and we get that

$$
\begin{aligned}
\int_{0}^{1}\left(\int_{x+y}^{x+y+1}\left\{\frac{1}{t}\right\}^{m} d t\right) d x= & \left.\left(x \int_{x+y}^{x+y+1}\left\{\frac{1}{t}\right\}^{m} d t\right)\right|_{x=0} ^{x=1} \\
& -\int_{0}^{1} x\left(\left\{\frac{1}{1+x+y}\right\}^{m}-\left\{\frac{1}{x+y}\right\}^{m}\right) d x \\
= & \int_{1+y}^{2+y} \frac{1}{t^{m}} d t-\int_{0}^{1} \frac{x}{(1+x+y)^{m}} d x \\
& +\int_{0}^{1} x\left\{\frac{1}{x+y}\right\}^{m} d x
\end{aligned}
$$


Thus,

$$
\begin{aligned}
I_{m}= & \int_{0}^{1}\left(\int_{1+y}^{2+y} \frac{1}{t^{m}} d t\right) d y-\int_{0}^{1} \int_{0}^{1} \frac{x}{(1+x+y)^{m}} d x d y \\
& +\int_{0}^{1} \int_{0}^{1} x\left\{\frac{1}{x+y}\right\}^{m} d x d y \\
= & \int_{0}^{1}\left(\int_{1+y}^{2+y} \frac{1}{t^{m}} d t\right) d y-\int_{0}^{1} \int_{0}^{1} \frac{x}{(1+x+y)^{m}} d x d y+\frac{1}{2} D_{1, m} .
\end{aligned}
$$

We distinguish the following cases.

Case $m=1$. We have, based on (3.1) and Corollary 2, that

$$
\begin{aligned}
I_{1} & =\int_{0}^{1} \ln \left(\frac{2+y}{1+y}\right) d y-\int_{0}^{1} \int_{0}^{1} \frac{x}{1+x+y} d x d y+\frac{1}{2} D_{1,1} \\
& =\frac{9 \ln 3}{2}-6 \ln 2-\frac{\zeta(3)}{6} .
\end{aligned}
$$

Case $m=2$. A calculation, based on (3.1) and part a) of Theorem 2, shows that

$$
\begin{aligned}
I_{2} & =\ln \frac{4}{3}-\int_{0}^{1} \int_{0}^{1} \frac{x}{(1+x+y)^{2}} d x d y+\frac{1}{2} D_{1,2} \\
& =6 \ln 2-3 \ln 3-\frac{\zeta(2)+\zeta(3)}{6} .
\end{aligned}
$$

Case $m=3$. We have, based on (3.1) and part b) of Theorem 2, that

$$
\begin{aligned}
I_{3} & =\frac{1}{6}-\int_{0}^{1} \int_{0}^{1} \frac{x}{(1+x+y)^{3}} d x d y+\frac{1}{2} D_{1,3} \\
& =\frac{\ln 3}{2}-\frac{3 \ln 2}{2}+\frac{5}{3}-\frac{\gamma}{2}-\frac{\zeta(2)}{4}-\frac{\zeta(3)}{6} .
\end{aligned}
$$

Case $m \geq 4$. A calculation, based on (3.1), shows that

$$
I_{m}=\frac{(7-m) \cdot 2^{2-m}+m-4-3^{3-m}}{(m-1)(m-2)(m-3)}+\frac{1}{2} D_{1, m},
$$

and the theorem is proved.

Remark 1. It is worth mentioning that this integral is recorded in [5] as the second part of Theorem 8. However, Qin and Lu have skipped the calculations of $I_{m}$ and instead they calculated a double integral and explained how the triple integral can be calculated by analogy. It turns out that the values of $I_{m}$ when $m=1,2$ and 3, as given by Qin and $\mathrm{Lu}$, are incorrect and by our method, which is different than the one mentioned in [5], these values are corrected. 


\section{A SPECIAL CLASS OF INTEGRALS AND A RECURRENCE FORMULA}

In this section we consider the multiple integral

$$
M_{m}^{n}=\int_{0}^{1} \cdots \int_{0}^{1}\left(x_{1}+x_{2}+\cdots+x_{n}\right)^{m}\left\{\frac{1}{x_{1}+\cdots+x_{n}}\right\} d x_{1} \cdots d x_{n},
$$

where $n \geq 2$ and $m \geq 0$ are integers, and we show that this integral can be calculated via a recurrence formula. The main result of the section is the following theorem.

Theorem 4. Let $n \geq 2$ and $m \geq 0$ be integers. The following recurrence formulas hold

$$
\begin{aligned}
M_{0}^{n}= & \frac{1}{n-1} M_{1}^{n-1}+\int_{0}^{1} \cdots \int_{0}^{1} \ln \left(1+x_{1}+\cdots+x_{n-1}\right) d x_{1} \cdots d x_{n-1} \\
& -\int_{0}^{1} \cdots \int_{0}^{1} \ln \left(1+x_{1}+\cdots+x_{n-2}\right) d x_{1} \cdots d x_{n-2}
\end{aligned}
$$

and, for $m \geq 1$,

$$
\begin{aligned}
M_{m}^{n}= & \frac{1}{n-1} M_{m+1}^{n-1}+\frac{1}{m} \int_{0}^{1} \cdots \int_{0}^{1}\left(1+x_{1}+\cdots+x_{n-1}\right)^{m} d x_{1} \cdots d x_{n-1} \\
& -\frac{1}{m} \int_{0}^{1} \cdots \int_{0}^{1}\left(1+x_{1}+\cdots+x_{n-2}\right)^{m} d x_{1} \cdots d x_{n-2} .
\end{aligned}
$$

Proof. We discuss only the case when $m \geq 1$ since the case when $m=0$ is handled similarly. We have, in view of the substitution $x_{1}+\cdots+x_{n}=y$, that

$$
\begin{aligned}
M_{m}^{n} & =\int_{0}^{1} \cdots \int_{0}^{1}\left(x_{1}+x_{2}+\cdots+x_{n}\right)^{m}\left\{\frac{1}{x_{1}+\cdots+x_{n}}\right\} d x_{1} \cdots d x_{n} \\
& =\int_{0}^{1} \cdots \int_{0}^{1}\left(\int_{x_{1}+\cdots+x_{n-1}}^{1+x_{1}+\cdots+x_{n-1}} y^{m}\left\{\frac{1}{y}\right\} d y\right) d x_{1} \cdots d x_{n-1} \\
& =\int_{0}^{1}\left(\int_{0}^{1}\left(\cdots \int_{0}^{1}\left(\int_{x_{1}+\cdots+x_{n-1}}^{1+\cdots+x_{n-1}} y^{m}\left\{\frac{1}{y}\right\} d y\right) d x_{n-1} \cdots\right) d x_{2}\right) d x_{1} .
\end{aligned}
$$

Since $\int_{x_{1}+\cdots+x_{n-1}}^{1+x_{1}+\cdots+x_{n-1}}=\int_{0}^{1}+\int_{1}^{1+x_{1}+\cdots+x_{n-1}}-\int_{0}^{x_{1}+\cdots+x_{n-1}}$, we have

$$
\begin{aligned}
& \int_{x_{1}+\cdots+x_{n-1}}^{1+x_{1}+\cdots+x_{n-1}} y^{m}\left\{\frac{1}{y}\right\} d y \\
& =\int_{0}^{1} y^{m}\left\{\frac{1}{y}\right\} d y+\int_{1}^{1+x_{1}+\cdots+x_{n-1}} y^{m}\left\{\frac{1}{y}\right\} d y-\int_{0}^{x_{1}+\cdots+x_{n-1}} y^{m}\left\{\frac{1}{y}\right\} d y \\
& =\int_{0}^{1} y^{m}\left\{\frac{1}{y}\right\} d y+\frac{\left(1+x_{1}+\cdots+x_{n-1}\right)^{m}-1}{m}-\int_{0}^{x_{1}+\cdots+x_{n-1}} y^{m}\left\{\frac{1}{y}\right\} d y,
\end{aligned}
$$


and it follows that

$$
\begin{aligned}
& \int_{0}^{1}\left(\int_{x_{1}+\cdots+x_{n-1}}^{1+x_{1}+\cdots+x_{n-1}} y^{m}\left\{\frac{1}{y}\right\} d y\right) d x_{n-1}=\int_{0}^{1} y^{m}\left\{\frac{1}{y}\right\} d y \\
& +\int_{0}^{1} \frac{\left(1+x_{1}+\cdots+x_{n-1}\right)^{m}-1}{m} d x_{n-1} \\
& \quad-\int_{0}^{1}\left(\int_{0}^{x_{1}+\cdots+x_{n-1}} y^{m}\left\{\frac{1}{y}\right\} d y\right) d x_{n-1} .
\end{aligned}
$$

We calculate the integral

$$
\int_{0}^{1}\left(\int_{0}^{x_{1}+\cdots+x_{n-1}} y^{m}\left\{\frac{1}{y}\right\} d y\right) d x_{n-1}
$$

by parts with

$$
\begin{aligned}
& f\left(x_{n-1}\right)=\int_{0}^{x_{1}+\cdots+x_{n-1}} y^{m}\left\{\frac{1}{y}\right\} d y, \\
& f^{\prime}\left(x_{n-1}\right)=\left(x_{1}+\cdots+x_{n-1}\right)^{m}\left\{\frac{1}{x_{1}+\cdots+x_{n-1}}\right\} \\
& g^{\prime}\left(x_{n-1}\right)=1, g\left(x_{n-1}\right)=x_{n-1}, \text { and we get that } \\
& \int_{0}^{1}\left(\int_{0}^{x_{1}+\cdots+x_{n-1}} y^{m}\left\{\frac{1}{y}\right\} d y\right) d x_{n-1} \\
&=\left.\left(x_{n-1} \int_{0}^{x_{1}+\cdots+x_{n-1}} y^{m}\left\{\frac{1}{y}\right\} d y\right)\right|_{x_{n-1}=0} ^{x_{n-1}=1} \\
&-\int_{0}^{1} x_{n-1}\left(x_{1}+\cdots+x_{n-1}\right)^{m}\left\{\frac{1}{x_{1}+\cdots+x_{n-1}}\right\} d x_{n-1} \\
&=\int_{0}^{1+x_{1}+\cdots+x_{n-2}} y^{m}\left\{\frac{1}{y}\right\} d y \\
&-\int_{0}^{1} x_{n-1}\left(x_{1}+\cdots+x_{n-1}\right)^{m}\left\{\frac{1}{x_{1}+\cdots+x_{n-1}}\right\} d x_{n-1} \\
&=\left.\int_{0}^{1} y^{m}\left\{\frac{1}{y}\right\} d y+\int_{1}^{1+x_{1}+\cdots+x_{n-2}} \frac{1}{y^{m}}\right\} d y \\
&-\int_{0}^{1} x_{n-1}\left(x_{1}+\cdots+x_{n-1}\right)^{m}\left\{\frac{1}{x_{1}+\cdots+x_{n-1}}\right\} d x_{n-1} \\
&= \int_{0}^{1} y^{m}\left\{\frac{1}{y}\right\} d y+\frac{\left(1+x_{1}+x_{2}+\cdots+x_{n-2}\right)^{m}-1}{m} \\
&-\int_{0}^{1} x_{n-1}\left(x_{1}+\cdots+x_{n-1}\right)^{m}\left\{\frac{1}{x_{1}+\cdots+x_{n-1}}\right\} d x_{n-1} .
\end{aligned}
$$


Thus,

$$
\begin{aligned}
& \int_{0}^{1}\left(\int_{x_{1}+\cdots+x_{n-1}}^{1+x_{1}+\cdots+x_{n-1}} y^{m}\left\{\frac{1}{y}\right\} d y\right) d x_{n-1} \\
& =\int_{0}^{1} \frac{\left(1+x_{1}+\cdots+x_{n-1}\right)^{m}}{m} d x_{n-1} \\
& \quad-\frac{\left(1+x_{1}+\cdots+x_{n-2}\right)^{m}}{m} \\
& \quad+\int_{0}^{1} x_{n-1}\left(x_{1}+\cdots+x_{n-1}\right)^{m}\left\{\frac{1}{x_{1}+\cdots+x_{n-1}}\right\} d x_{n-1} .
\end{aligned}
$$

Integrating with respect to variables $x_{1}, \ldots, x_{n-2}$, we get that

$$
\begin{aligned}
& M_{m}^{n}=\int_{0}^{1} \cdots \int_{0}^{1} \frac{\left(1+x_{1}+\cdots+x_{n-1}\right)^{m}}{m} d x_{1} \cdots d x_{n-1} \\
& -\int_{0}^{1} \cdots \int_{0}^{1} \frac{\left(1+x_{1}+\cdots+x_{n-2}\right)^{m}}{m} d x_{1} \cdots d x_{n-2} \\
& +\int_{0}^{1} \cdots \int_{0}^{1} x_{n-1}\left(x_{1}+\cdots+x_{n-1}\right)^{m}\left\{\frac{1}{x_{1}+\cdots+x_{n-1}}\right\} d x_{1} \cdots d x_{n-1} .
\end{aligned}
$$

By symmetry, for all $i, j=1, \ldots, n-1$, one has that

$$
\begin{aligned}
& \int_{0}^{1} \cdots \int_{0}^{1} x_{i}\left(x_{1}+\cdots+x_{n-1}\right)^{m}\left\{\frac{1}{x_{1}+\cdots+x_{n-1}}\right\} d x_{1} \cdots d x_{n-1} \\
& =\int_{0}^{1} \cdots \int_{0}^{1} x_{j}\left(x_{1}+\cdots+x_{n-1}\right)^{m}\left\{\frac{1}{x_{1}+\cdots+x_{n-1}}\right\} d x_{1} \cdots d x_{n-1},
\end{aligned}
$$

and hence

$$
\begin{aligned}
\int_{0}^{1} & \cdots \int_{0}^{1} x_{n-1}\left(x_{1}+\cdots+x_{n-1}\right)^{m}\left\{\frac{1}{x_{1}+\cdots+x_{n-1}}\right\} d x_{1} \cdots d x_{n-1} \\
& =\frac{1}{n-1} \int_{0}^{1} \cdots \int_{0}^{1}\left(x_{1}+\cdots+x_{n-1}\right)^{m+1}\left\{\frac{1}{x_{1}+\cdots+x_{n-1}}\right\} d x_{1} \cdots d x_{n-1} .
\end{aligned}
$$

It follows that

$$
\begin{aligned}
M_{m}^{n}= & \frac{1}{n-1} M_{m+1}^{n-1}+\frac{1}{m} \int_{0}^{1} \cdots \int_{0}^{1}\left(1+x_{1}+\cdots+x_{n-1}\right)^{m} d x_{1} \cdots d x_{n-1} \\
& -\frac{1}{m} \int_{0}^{1} \cdots \int_{0}^{1}\left(1+x_{1}+\cdots+x_{n-2}\right)^{m} d x_{1} \cdots d x_{n-2} .
\end{aligned}
$$

Integrals of the form $\int_{0}^{1} \cdots \int_{0}^{1}\left(1+x_{1}+\cdots+x_{k}\right)^{m} d x_{1} \cdots d x_{k}$ are calculated by using the multinomial formula. The case when $m=0$ follows by a similar argument. The theorem is proved. 
Now we show how this recurrence formulas can be used for establishing the integral equalities

$$
M_{0}^{3}=\int_{0}^{1} \int_{0}^{1} \int_{0}^{1}\left\{\frac{1}{x+y+z}\right\} d x d y d z=\frac{9}{2} \ln 3-6 \ln 2-\frac{\zeta(3)}{6}
$$

and

$$
\begin{aligned}
M_{1}^{3} & =\int_{0}^{1} \int_{0}^{1} \int_{0}^{1}(x+y+z)\left\{\frac{1}{x+y+z}\right\} d x d y d z \\
& =\frac{5}{6}+\frac{1}{48} \sum_{j=1}^{\infty}(2+j)(3+j)(\zeta(j+4)-1) .
\end{aligned}
$$

We have, based on Theorem 4 with $m=0$ and $n=3$, that

$$
\begin{aligned}
M_{0}^{3} & =\frac{1}{2} M_{1}^{2}+\int_{0}^{1} \int_{0}^{1} \ln (1+x+y) d x d y-\int_{0}^{1} \ln (1+x) d x \\
& =\frac{1}{2} D_{1,1}+\frac{1}{2}(9 \ln 3-8 \ln 2-3)-(2 \ln 2-1) \\
& =\frac{9}{2} \ln 3-6 \ln 2-\frac{\zeta(3)}{6} .
\end{aligned}
$$

On the other hand,

$$
\begin{aligned}
M_{1}^{3} & =\frac{1}{2} M_{2}^{2}+\int_{0}^{1} \int_{0}^{1}(1+x+y) d x d y-\int_{0}^{1}(1+x) d x \\
& =\frac{1}{2} D_{2,1}+\frac{1}{2}=\frac{1}{3}+\frac{1}{2} V_{1,3} \\
& =\frac{5}{6}+\frac{1}{48} \sum_{j=1}^{\infty}(2+j)(3+j)(\zeta(j+4)-1),
\end{aligned}
$$

where the last equality follows from Theorem 1 with $k=1$ and $m=3$.

\section{REFERENCES}

[1] O. Furdui, "Problem 150, Problems," Missouri J. Math. Sci., vol. 16, no. 2, 2004.

[2] O. Furdui, "Exotic fractional part integrals and Euler's constant," Analysis, vol. 31, pp. 249-257, 2011, doi: 10.1524/anly.2011.1131.

[3] J. Havil, Gamma. Exploring Euler's constant. Princeton and Oxford: Princeton University Press, 2003.

[4] H. Qin, "Problem 150, Solutions," Missouri J. Math. Sci., vol. 17, no. 3, 2005.

[5] H. Qin and Y. Lu, "Integrals of fractional parts and some new identities on Bernoulli numbers," Int. J. Contemp. Math. Sciences, vol. 6, no. 15, pp. 745-761, 2011. 
Author's address

\section{Ovidiu Furdui}

Technical University of Cluj-Napoca, Department of Mathematics, Str. Memorandumului Nr. 28, 400114 Cluj-Napoca, Romania

E-mail address: Ovidiu.Furdui@math.utcluj.ro 